Research Article

\title{
Seroconversion and Abundance of IgG Antibodies against S1-RBD of SARS-CoV-2 and Neutralizing Activity in the Chilean Population
}

\author{
R. González-Stegmaier, ${ }^{1}$ K. Cereceda, ${ }^{1}$ J. L. Briones, ${ }^{2}$ C. Beltran-Pávez, ${ }^{3,4}$ \\ A. Oyarzún-Arrau, ${ }^{3,4}$ S. Riquelme-Barrios, ${ }^{3,4}$ C. Selman, ${ }^{5,6}$ F. Yarad, ${ }^{5}$ M. Mahave, ${ }^{7}$ \\ C. Caglevic, ${ }^{8}$ R. Morales, ${ }^{9}$ A. Aguirre, ${ }^{1}$ F. Valiente-Echeverría, ${ }^{3,4}$ R. Soto-Rifo, ${ }^{3,4}$ \\ H. Marsiglia, ${ }^{10}$ R. Gazitua $\mathbb{D}^{2},{ }^{2}$ and F. Villarroel-Espindola $\mathbb{D}^{1,8}$ \\ ${ }^{1}$ Translational Medicine Laboratory, Instituto Oncológico Fundación Arturo López Pérez, Santiago, Chile \\ ${ }^{2}$ Haematology Department, Instituto Oncológico Fundación Arturo López Pérez, Santiago, Chile \\ ${ }^{3}$ Laboratory of Molecular and Cellular Virology, Virology Program, Institute of Biomedical Sciences, Faculty of Medicine, \\ Universidad de Chile, Chile \\ ${ }^{4}$ HIVIAIDS Work Group, Faculty of Medicine, Universidad de Chile, Chile \\ ${ }^{5}$ Diagnostic Units, Instituto Oncológico Fundación Arturo López Pérez, Santiago, Chile \\ ${ }^{6}$ Biobank, Instituto Oncológico Fundación Arturo López Pérez, Santiago, Chile \\ ${ }^{7}$ Medical Oncology Department, Instituto Oncológico Fundación Arturo López Pérez, Santiago, Chile \\ ${ }^{8}$ Cancer Research Department, Instituto Oncológico Fundación Arturo López Pérez, Santiago, Chile \\ ${ }^{9}$ Internal Medicine Department, Instituto Oncológico Fundación Arturo López Pérez, Santiago, Chile \\ ${ }^{10}$ Radiotherapy Department, Instituto Oncológico Fundación Arturo López Pérez, Santiago, Chile
}

Correspondence should be addressed to R. Gazitua; raimundo.gazitua@falp.org and F. Villarroel-Espindola; franz.villarroel@falp.org

Received 20 December 2020; Revised 3 January 2021; Accepted 24 January 2021; Published 23 February 2021

Academic Editor: Baohui Xu

Copyright @ 2021 R. González-Stegmaier et al. This is an open access article distributed under the Creative Commons Attribution License, which permits unrestricted use, distribution, and reproduction in any medium, provided the original work is properly cited.

COVID-19 is a pandemic caused by SARS-CoV-2. In Chile, half a million people have been infected and more than 16,000 have died from COVID-19. As part of the clinical trial NCT04384588, we quantified IgG against S1-RBD of SARS-CoV-2 (anti-RBD) in recovered people in Santiago and evaluated their suitability as COVID-19 convalescent plasma donors. ELISA and a luminescent SARS-CoV-2 pseudotype were used for IgG and neutralizing antibody quantification. $72.9 \%$ of the convalescent population (468 of 639) showed seroconversion $(5-55 \mu \mathrm{g} / \mathrm{mL}$ anti-RBD IgG) and were suitable candidates for plasma donation. Analysis by gender, age, and days after symptom offset did not show significant differences. Neutralizing activity correlated with an increased concentration of anti-RBD IgG $(p<0.0001)$ and showed a high variability between donors. We confirmed that the majority of the Chilean patients have developed anti-SARS-CoV-2 antibodies. The quantification of anti-RBD IgG in convalescent plasma donors is necessary to increase the detection of neutralizing antibodies.

\section{Introduction}

Coronavirus disease 2019 (COVID-19) is caused by severe acute respiratory syndrome coronavirus 2 (SARS-CoV-2). It emerged in December 2019 in Wuhan, China $[1,2]$, and was subsequently declared a worldwide pandemic by the World Health Organization (WHO) [3]. Currently, Chile has reported half a million infected people and more than 16,000 deaths caused by COVID-19. The government and the private sector have implemented several programs to 
mitigate this pandemic, including PCR and rapid testing across the country, while some institutions have started trials for convalescent plasma. However, these efforts still do not seem to be enough to control this sanitary emergency.

The measurement of serum antibodies has provided crucial data for understanding key aspects of the infection [4]. It is widely accepted that IgM provides the first line of defense during viral infections, before generating an adaptive response with a high-affinity IgG production, which is important for long-term immunity and immunological memory [5]. In patients infected with SARS-CoV-2, immunoglobulin M (IgM) antibodies are detectable around 7 days postinfection and IgG antibodies usually take two weeks to develop [6-9]. The clinical features of COVID-19 vary widely, ranging from asymptomatic to mild or severe forms with multiorgan dysfunction, and most COVID-19 data were derived from hospitalized patients [8, 10-13].

Accurate quantitative measurement of anti-SARS-CoV-2 antibody responses is essential for public health interventions and therapeutic applications $[14,15]$. The SARS-CoV-2 shows two structural proteins as major immunogens, the spike $(\mathrm{S})$ and nucleocapsid $(\mathrm{N})$ proteins, with the RBD domain of the $S$ protein being responsible for the infection of respiratory epithelial cells via interaction with the cell surface receptor angiotensin converting enzyme 2 (ACE2). Antibodies against this segment have been reported as antiviral based on their neutralizing activity in plasma $[7,13,16-$ 18]. In this study, we quantified the abundance of $\mathrm{IgG}$ antibodies against the S1-RBD fragment of SARS-CoV-2 in people who recovered from COVID-19 in Santiago, Chile. After analyzing 639 convalescent participants, $72.9 \%$ showed anti-RBD IgG seroconversion with a concentration range in serum between 5 and $55 \mu \mathrm{g} / \mathrm{mL}$, and no significant correlation with gender, age, and days after symptom offset were observed between groups. Further analysis showed that serum samples with antibodies above $20.9 \mu \mathrm{g} / \mathrm{mL}$ showed a statistically increased proportion of neutralizing antibodies (Nab) against SARS-CoV-2 during convalescence. Regarding early seroconversion, 14 of 26 cases within the first two weeks of severe COVID-19 showed positive levels of anti-RBD IgG with a mean of $21.2 \mu \mathrm{g} / \mathrm{mL}$, and 7 cases were above the 75 th percentile. Our results confirmed a heterogeneous but specific IgG seroconversion after SARS-CoV-2 infection within the population from the metropolitan area of Chile. The production of antibodies against the RBD of spike were shown to be as early as the first 14 days after symptoms started, and those can persist in the blood for almost two months and display protective neutralizing activity during active disease and convalescence.

\section{Materials and Methods}

2.1. Patients and Samples. This research consisted of 690 samples distributed in 3 groups: 639 convalescents, 26 active COVID-19 cases, and 25 healthy never-exposed people as a negative control (supplementary Table 1). There were a second cohort of 72 convalescent plasma donors for the analysis of neutralizing antibodies and a third cohort of 100 unrelated cases (positive and negative COVID-19) for
ELISA validation. Recovered volunteers and convalescent plasma donors met all criteria for the clinical trial NCT04384588 and signed the specific consent letter. All volunteer patients who recovered from COVID-19 were asymptomatic for at least 21 days and showed a negative result for SARS-CoV-2 by real-time polymerase chain reaction (PCR) test. Volunteers with active COVID-19 consented at the moment of hospitalization and met the following inclusion criteria: (a) patients over 18 years old and (b) COVID-19 diagnosis at enrolment confirmed with a positive SARS-CoV-2 PCR in a nasopharyngeal swab.

All SARS-CoV-2-related samples used in this research were collected between July and August 2020, in Santiago, Chile.

Healthy group samples were considered as plasma from a regular blood donation obtained before October 2019 (neverexposed cases) and as a single blood sample drawn in April 2020 from asymptomatic healthy volunteers with a negative SARS-CoV-2 PCR result (contemporary cases).

For the active cases, treatments given before blood collection are described in supplemental Table 2.

2.2. Quantitative IgG ELISA Anti-RBD S1 SARS-CoV-2. AntiSARS-CoV-2 IgG ELISA test was developed and validated in the Translational Medicine Laboratory of Fundación Arturo López Pérez (Cereceda et al., submitted); a comparative table (Table S3) is included as a supplementary data. Briefly, MaxiSorp $^{\text {TM }}$ 96-well microplates (439454, NUNC, Thermo Fisher Scientific) were coated with $50 \mathrm{ng}$ of S1-RBD protein of SARS-CoV-2 (RB.230-30162-100, RayBiotech) in $0.1 \mathrm{M}$ carbonate buffer $\mathrm{pH} 9$ and incubated overnight at $4^{\circ} \mathrm{C}$. The coating solution was removed and each well was washed once with cold phosphate-buffered saline solution (PBS). An uncoated surface was blocked for 4 hours at room temperature using $400 \mu \mathrm{L}$ of blocking solution containing $5 \%$ skim milk in PBS pH7 and supplemented with $0.1 \%$ bovine serum albumin (BSA), 0.1\% nonimmune donkey serum (017-000-121, Jackson ImmunoResearch), and 0.1\% Tween-20. Later, the solution was eliminated and the plates were air dried before storing and freezing at $-20^{\circ} \mathrm{C}$. Frozen plates were shown to be stable for up to 45 days based on the measurement of the interwell variation coefficient of blanks and positive controls. Before testing the unknown samples, the plates were thawed at room temperature and the excess blocking solution was removed using a washing solution (0.1\% Tween-20 in PBS). Serum samples, controls, and calibrators were diluted fresh at 1:320 using a solution containing $0.1 \%$ BSA in PBS supplemented with $0.1 \%$ nonimmune donkey serum and $0.1 \%$ Tween- 20 . The calibration curve was prepared by serial dilution at a factor of 10 of a commercial chimeric mouse $\mathrm{scFv}$ fused with human IgG1 Fc anti-SARS-CoV-2-S1-RBD (CSBYP3324GMY1, Cusabio). $100 \mu \mathrm{L}$ of each dilution was seeded in duplicate and incubated for 1 hour at room temperature $\left(20 \pm 2^{\circ} \mathrm{C}\right)$. Then, all liquids were removed and the plate rinsed 5 times with $250 \mu \mathrm{L}$ of washing solution with $2 \mathrm{sec}$ shaking and $10 \mathrm{sec}$ soaking each time in an automatic microplate washer. 
Later, each well was incubated at $20^{\circ} \mathrm{C}$ for $1 \mathrm{~h}$ with $100 \mu \mathrm{L}$ of $20 \mathrm{ng} / \mathrm{mL}$ solution of peroxidase-conjugated AffiniPure Donkey Anti-Human IgG, Fc $\gamma$ fragment specific (709-035098, Jackson ImmunoResearch). Finally, the assay was developed using 3,3',5,5' -tetramethyl-benzidine substrate (T0440, Sigma-Aldrich) and stopped after 15 minutes with $2 \mathrm{M}$ sulfuric acid; then, the absorbance was measured at $450 \mathrm{~nm}$ in a Cytation $5^{\circledR}$ plate reader (BioTek). For interpretation, we have the following: (1) qualitative absorbance ratio between the unknown sample and a calibrator (positive ratio $>1.1$ ;negative ratio $<0.9$; undetermined $1.1<$ ratio $>0.9$ ) and (2) quantitative interpolation from a standard calibration curve. Blank, controls, calibrators, and curves were performed within the respective $5-10 \%$ coefficient variation (CV).

Sera previously titered using an IVD ELISA test (Euroimmun) were used as internal positive controls (ratio above $2.5,7.5 \% \mathrm{CV}$ ), as a calibrator (ratio $1.0 \pm 0.05,<5 \% \mathrm{CV}$ ), and as internal negative controls (ratio below $0.8,5 \% \mathrm{CV}$ ). Additionally, a calibration curve between 0.0 and $250.0 \mathrm{ng} / \mathrm{mL}$ of specific IgG was used, and it included as a reference material a commercial chimeric mouse $s c F v$ fused with human IgG1 Fc anti-SARS-CoV-2-S1-RBD (CSB-YP3324GMY1, Cusabio).

2.2.1. Neutralization Assay. Samples were diluted in DMEM, and $50 \mu \mathrm{L}$ of dilution was added to each well in triplicate. Three to $5 \mathrm{pg}$ of a luciferase-coding SARS-CoV-2 pseudotype was prepared freshly, mixed 1:1 with a diluted sample, and incubated for 1 hour at $37^{\circ} \mathrm{C}$. DMEM alone was used as a positive control (100\% infectivity). Then, $1 \times 10^{4}$ of HEKACE2 cells were incubated with each sample mix and cultured for $48 \mathrm{~h}$ before measuring the firefly-luciferase activity. Neutralizing assays were validated considering the following pass/fail criteria: (1) The average relative light units (RLU) of the pseudovirus control wells are $\geq 10$ times the average RLU of the negative control wells (HEK293T cells). (2) The coefficient of variation (CV) between RLU in the pseudotype control wells is $\leq 30 \%$. (3) The percentage difference for triplicate wells is $\leq 30 \%$ for sample dilutions that yield at least $40 \%$ neutralization. (4) Positive control neutralization curve crosses the $50 \%$ neutralization cut-off $0-1$ times. (5) Finally, sigmoid curves and estimation of infectious dose 80 (ID80) were obtained using a 4-parameter nonlinear regression curve fit [19].

2.3. Statistical Analyses. Statistical analysis was performed using GraphPad Prism software, version 8.0. Qualitative and quantitative correlation was assessed using Pearson's correlation coefficient. Fisher's contingency test was used to assess the IgG levels and neutralization activity. Differences in mean values between groups were analyzed by the Mann-Whitney test. All values were depicted as a geometric mean with 95\% CI. One-way ANOVA and Tukey's multiple comparison test were performed for statistical analysis between various variables. The critical value for statistical significance was established as $p \leq 0.05$. Values marked with asterisks mean the following: ${ }^{*} p<0.05,{ }^{* *} p<0.01$, ${ }^{* * *} p<0.001$, and ${ }^{* * * *} p<0.0001$.

\section{Results}

3.1. Objective Metrics for Stratification and Plasma Donor Selection Based on Anti-RBD IgG Levels. For this research, we developed and validated a qualitative ELISA for the detection of a human IgG anti-S1-RBD fragment of SARS-CoV-2 (anti-RBD IgG). Previously, a qualitative ELISA from our laboratory showed $99 \%$ sensitivity and $85 \%$ specificity, and a percentage of agreement of 92.1 compared to the IVD ELISA from Euroimmun (Cereceda et al., 2020, submitted). For all our analyses, each plate used was coated with $50 \mathrm{ng}$ of antigen in a proportion of $1.8 \mathrm{pmol}$ of S1-RBD fragment per cubic centimeter of active surface. Using a commercial chimeric mouse scFv fused with human IgG1 Fc antiSARS-CoV-2-S1-RBD (CSB-YP3324GMY1, Cusabio) as reference material, our improved ELISA showed a linearity between 6.25 and $50 \mu \mathrm{g} / \mathrm{mL}$, a limit of detection (LoD) of $6 \mathrm{ng} / \mathrm{mL}$, and $40 \mathrm{ng} / \mathrm{mL}$ as limit of quantification (LoQ) for the specific IgG. This assay showed for the qualitative and quantitative analyses a direct proportionality and correlation with Pearson's $r$ coefficient of 0.9916 with a 95\% CI from 0.9523 to 0.9985 and apvalue $<0.0001$, allowing both metrics for sample analysis (Supplemental Figure S1). Using 100 samples previously tested for anti-SARS-CoV-2 IgG, the assay showed $99 \%$ specificity and $98.5 \%$ sensitivity, and a correlation of $92 \%$. Based on the covariance observed in a local group never exposed to SARS-CoV-2 (mean $2.4 \mu \mathrm{g} / \mathrm{mL}$ ), the cut-off for positivity was estimated using the mean plus 3 standard deviation (mean $+3 \mathrm{SD})$ to consider $99.73 \%$ of the negative population in a normal distribution (Supplemental Figure S1), and the calculated value was $6.6 \mu \mathrm{g} / \mathrm{mL}$ of anti-RBD IgG in serum.

\subsection{Anti-RBD IgG Seroconversion in Individuals Exposed to} SARS-CoV-2 in Santiago, Chile. 639 serum samples were collected in Santiago, Chile, during July and August 2020, and were analyzed to estimate the seroconversion and quantification of specific anti-RBD IgG. This cohort included convalescent cases between 21 and 123 days after their symptoms were offset, with a median of 34 days (Supplemental Table 1). All patients recovered from mild to moderate COVID-19 without special medical requirements. Overall, the infection with SARS-CoV-2 promoted the specific production of antibodies in infected people, and their concentrations in blood were substantially and statistically higher than asymptomatic never-exposed people $(p<0.0001)$ (Figure 1(a)). In fact, the segregation of positive and negative cases using a quantitative cut-off showed a positivity of $72.9 \%$ (466 of 639 ), with a geometric mean of $14.84 \mu \mathrm{g} / \mathrm{mL}$ and a calculated 75th percentile (P75) of $20.9 \mu \mathrm{g} / \mathrm{mL}$ of antiRBD IgG in serum for that group; most of the positive cases showed a range between 5 and $20 \mu \mathrm{g} / \mathrm{mL}$ (Figure 1(b)). The convalescent population included in this study had a median age of 35 years old; when the levels of IgG were analyzed by participant's age, they showed a high dispersion with no significant differences between groups, except between the two extreme age groups (18-24 versus 55-plus years old) where higher levels of anti-RBD were observed for older people $(p=0.015)$ (Figure $1(c)$ ). Regarding the time after 


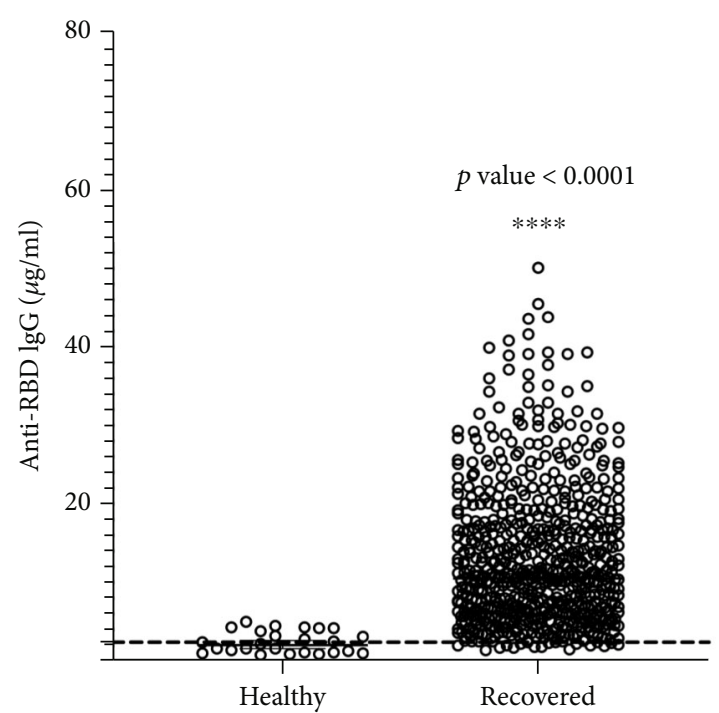

(a)

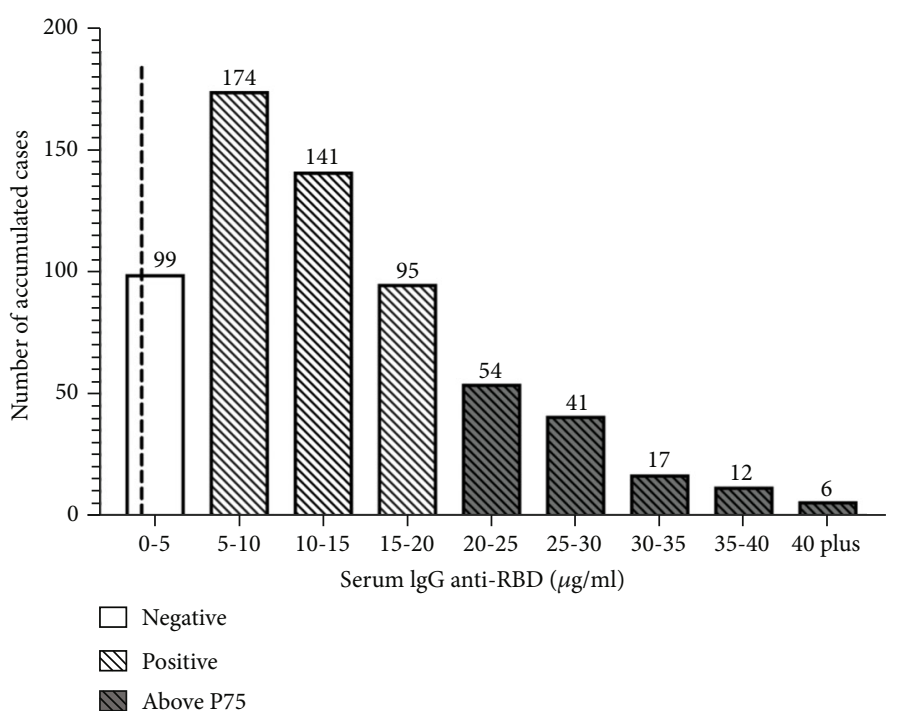

(b)

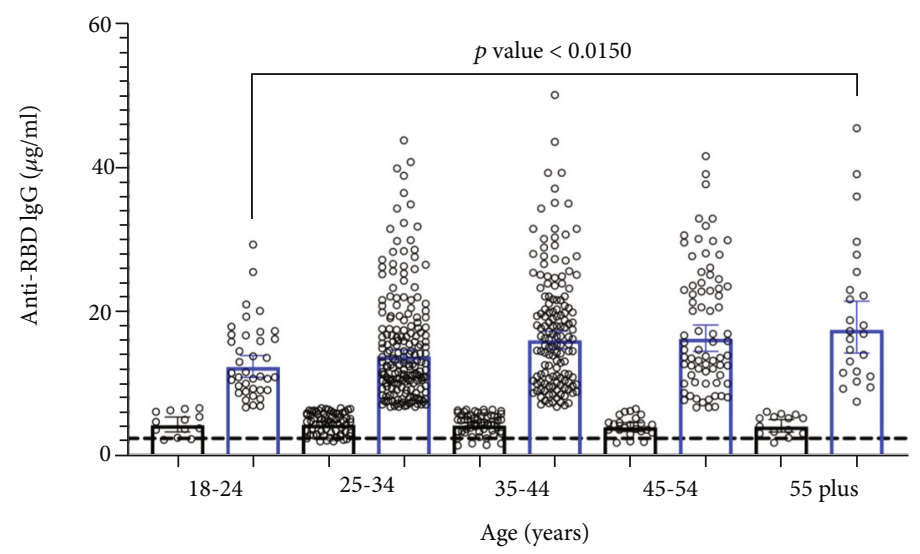

(c)

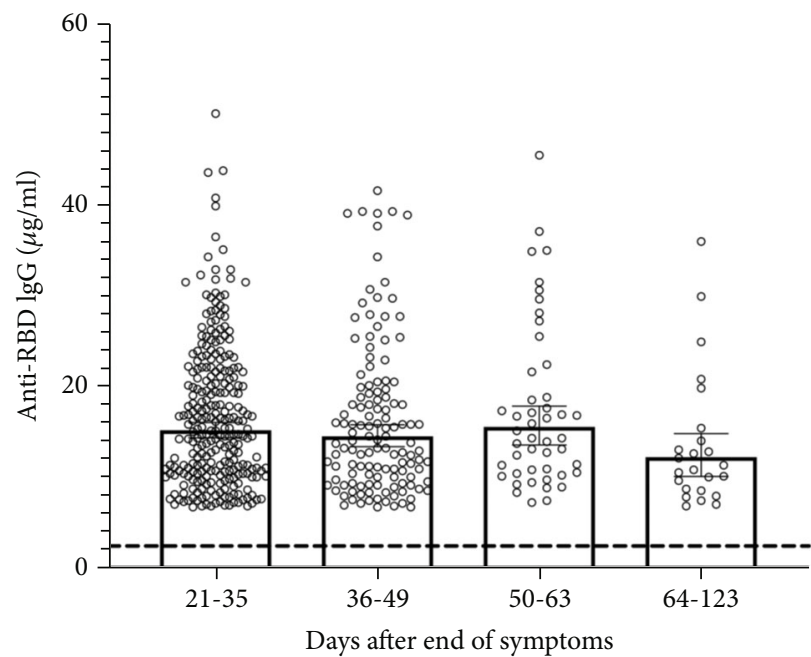

(d)

Figure 1: Analysis of anti-RBD IgG in individuals exposed to SARS-CoV-2 in the metropolitan region of Chile. (a) Anti-RBD IgG levels in participants, namely, healthy controls as the baseline group $(N=25)$, and participants who recovered from COVID-19 with different days of convalescence $(N=639)$. (b) Histogram for accumulated frequency and antibody concentration range. Positive and negative ELISA results are indicated. Darker bars represent the 75th percentile (P75) for the studied cohort. (c) Anti-RBD levels based on age for positive (blue bars) and negative (black bars) cases. (d) Levels of IgG after symptom offset in the convalescent group and positive qualitative ELISA. (ad) In all cases, the dashed line corresponds to the healthy controls. 
symptoms were offset, the global average concentration of anti-RBD IgG did not show any statistical differences between groups (Figure $1(\mathrm{~d})$ ). When the population was segregated by gender, no statistical differences were observed (Figures 2(a) and 2(b)); however, a trend of higher levels of specific IgG were observed for older men compared with the younger group $(p=0.0461)$ (Figure $2(b))$. The amounts of anti-RBD antibodies were not influenced by the time after symptoms ended and did not show any statistical differences between men or women groups (Figures 2(c) and 2(d)).

Regarding the 75th percentile (P75) by gender, men showed statistically higher levels of IgG than women when P75 was estimated for each independent gender group (high male vs. high female, $p=0.0030)$, and specifically 67 of 268 women $(25 \%)$ and 48 of 198 men $(24.2 \%)$ were above the global P75 with an average concentration of specific IgG of $26.5 \mu \mathrm{g} / \mathrm{mL}$ and $29.6 \mu \mathrm{g} / \mathrm{mL}$, respectively (Supplemental Figure S2).

3.3. Anti-RBD IgG Kinetic and Neutralization Activity in Convalescent Plasma. To evaluate the persistence of antibodies against SARS-CoV-2 after infection, six cases were under follow-up and evaluated for up to 50 days of convalescence, and the blood collection started 28 days after their symptoms finished. In addition, the neutralization activity in serum was measured as the $80 \%$ Inhibitory Dilution (ID) using pseudoviral particles [19]. The cases showed anti-RBD IgG levels below and above the measured P75 (concentration $=20.9 \mu \mathrm{g} / \mathrm{mL}$ ) but significantly higher than the control group, and a broad range of neutralizing ID80 between 83 and 12,400 (Figures 3(a) and 3(b)). Individuals with a similar IgG concentration exhibited dramatic differences in the ability to neutralize the virus in vitro, up to 100 -fold higher than others, and the starting amount of IgG did not reflect the observed decaying curve for the specific IgG or neutralizing activity (Figures 3(b) and $3(\mathrm{c}))$. Overall, the quantified concentration of antiRBD IgG correlated with the presence of SARS-CoV-2 $\mathrm{NAb}$ (Pearson's $r=0.51, p=0.001$ ) (data not shown). Further analysis considering 72 unrelated cases showed that serum samples with antibodies below P75 had statistically very low levels of neutralization $(p<0.0001)$ (Figure $3(d)$ ) and, when the patients were selected based on IgG above that cut-off, those showed a significantly increased proportion of NAb $(p<0.0001)$ (Figure 3(e)).

3.4. Early Seroconversion in Individuals with an Active Infection of SARS-CoV-2. We analyzed samples from 26 patients who were categorized as severe COVID-19 cases within the first 14 days of their diagnosis of an infection with SARS-CoV-2 (Table 1). This group showed $53.8 \%$ positivity (14 of 26) with a mean concentration of $21.2 \mu \mathrm{g} / \mathrm{mL}$ of antiRBD IgG, and 7 out of the 26 patients (27\%) had antibodies above the 75 th percentile $(20.9 \mu \mathrm{g} / \mathrm{mL})$ (Figure $4(\mathrm{a})$, red circle). We did not observe correlations in IgG levels and previous medication to the blood drawing (Table S2), and only one case received hydroxychloroquine as treatment of a systemic lupus erythematosus before the COVID-19 diagnosis. A contingency analysis showed a correlation between days of disease and seroconversion $(p=0.0053)$, increasing the frequency of positivity after 5 or more days after the symptoms started. However, 5 of 26 cases (19.2\%) developed antibodies against RBD within the first 5 days (Figure 4(b)). No correlation between age at diagnosis and early seroconversion was observed $(p>0.9999)$ (Figure 4(b)). As we expected, the presence of anti-RBD antibodies correlated with the ability to neutralize SARSCoV-2 in vitro $(p=0.0048)$, but the magnitude of the measured neutralizing activity in serum from patients with a severe COVID-19 was independent of the days after symptoms started $(p=0.2262)$ (Figure $4(b))$.

\section{Discussion}

Early results from the Hubei province of China showed a polyclonal IgG prevalence against SARS-CoV-2 of $89.8 \%$ (95\% CI $88.2-91.3 \%)$ in 1,740 COVID-19 convalescents [20], and the severity of the symptoms during COVID-19 have been related to the duration of humoral response, where asymptomatics or individuals with a mild illness have shown a quicker reduction in antibody titers than more severe cases $[21,22]$. In the current study, $50 \%$ of the convalescent population was considered within the range between 18 and 68 years old with a median age of 35 , which represents the main workforce in Chile. The estimated seroconversion was $72.9 \%$, which did not show significant differences at the level of gender, age, and days after symptom offset; however, the serum concentration of IgG anti-SARS-CoV-2 varied dramatically between cases, and it was not reflected in the measured neutralizing activity. Our observations were consistent with the previously reported seroconversion rates after four weeks of symptoms [20, 22, 23]. It is interesting that the Chilean convalescent population of this study showed similar results compared to a group of 49 recovered COVID-19 patients recruited in Wuhan, China, between February and March 2020 [24]. In 2020, Li et al. reported that 28 days after the onset of symptoms, $90 \%$ of the group (18-55 years old) showed an S-RBD-specific IgG titer above $1: 160$ and $78 \%$ had a titer of 1:640 or higher [24], which means that $90 \%$ of that group seroconverted after infection, and the S-RBDspecific and N-specific IgG antibodies increased after 4 weeks from the onset of symptoms, with no significant correlation to age, sex, or ABO blood type [24]. It is important to consider that an IgG response against the spike RBD domain has been associated with a patient's improved survival independently of other factors such as sex or age, supporting the concept that these antibodies are a significant contributor to the protective effect of humoral immunity in COVID-19 [25]. Additionally, we observed a significantly higher amount of anti-RBD IgG in older men, and it was previously shown in a preprint article where the authors suggested that being male, an older adult, and being hospitalized with COVID19 were each associated with having greater neutralizing antibody titers and IgGs against the S1-domain, the S1-RBD fragment, or the full spike protein [26].

Several authors have reported a rapid seroconversion during the first weeks of symptoms [23, 25, 27]; we observed $53.8 \%$ of IgG seroconversion in severe COVID-19 patients 


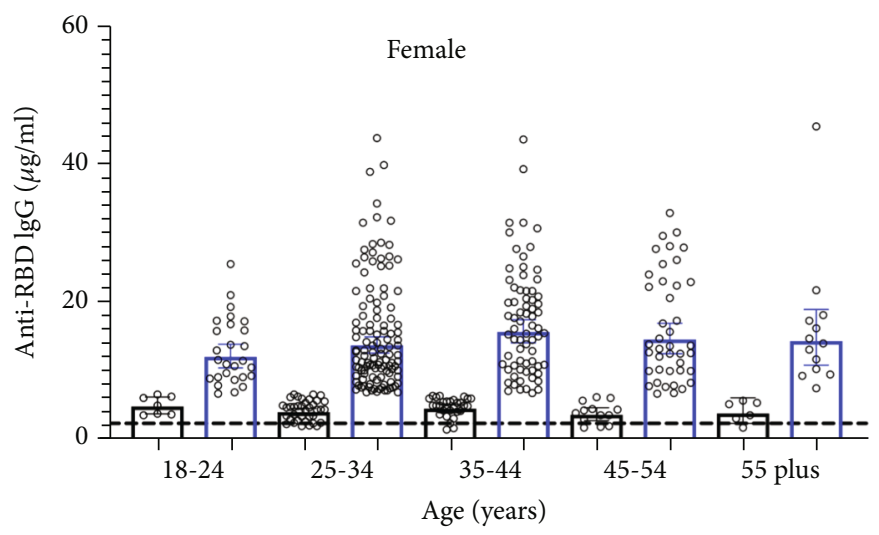

(a)

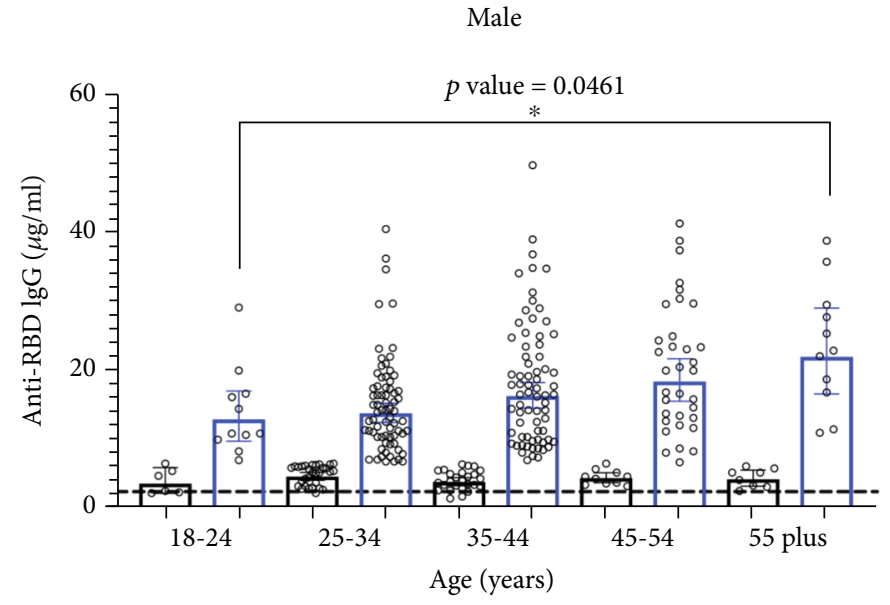

(b)

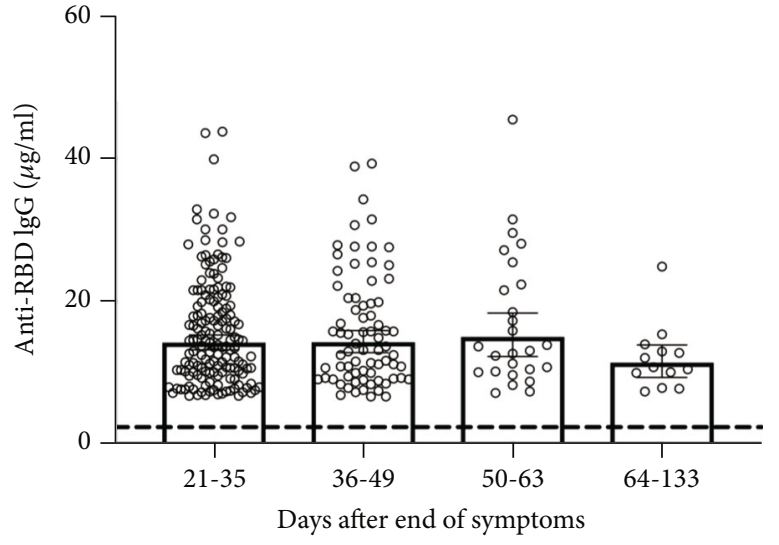

(c)

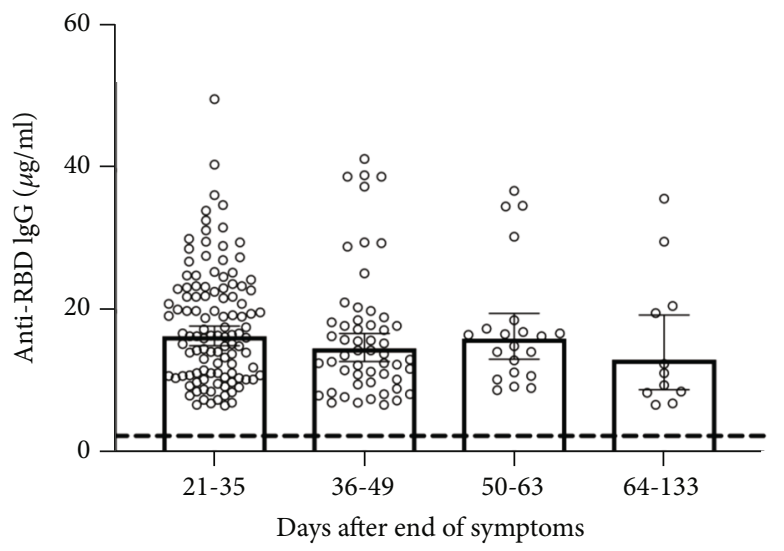

(d)

FIGURE 2: Stratified anti-RBD IgG levels by gender, age, and days of convalescence. Measured IgG distribution for positive (blue) and negative (black) quantitative ELISA. Cases were segregated as female $(N=361)(\mathrm{a}, \mathrm{c})$ and male $(N=278)(\mathrm{b}, \mathrm{d})$ based on age and days after symptom offset. In all cases, the dashed line corresponds to the control group.

between 4 and 9 days after a positive PCR test. A very early study reported for a small group (17) showed an anti-RBDspecific seroconversion rate of $64.71 \%$ for IgG within the 4 10 days after illness onset; however, after 15 days of disease, the seroconversion rate reached $100 \%$ (169 cases) for the three immunoglobulins [28]. Another study which considered 28 mild and 7 severe COVID-19 cases showed that
IgG in serum was detected in 1 of the 35 cases at the first week after the date of illness onset; however, at the third week, $68.9 \%(n=20 / 29)$ of the patients seroconverted in mild cases, while it was $100 \%(n=7 / 7)$ in severe cases [29]. Recently, it was reported from 166 studied cases that patients within the first week of symptoms with a very severe case of COVID-19 $(n=45)$ had a quicker and more significant 


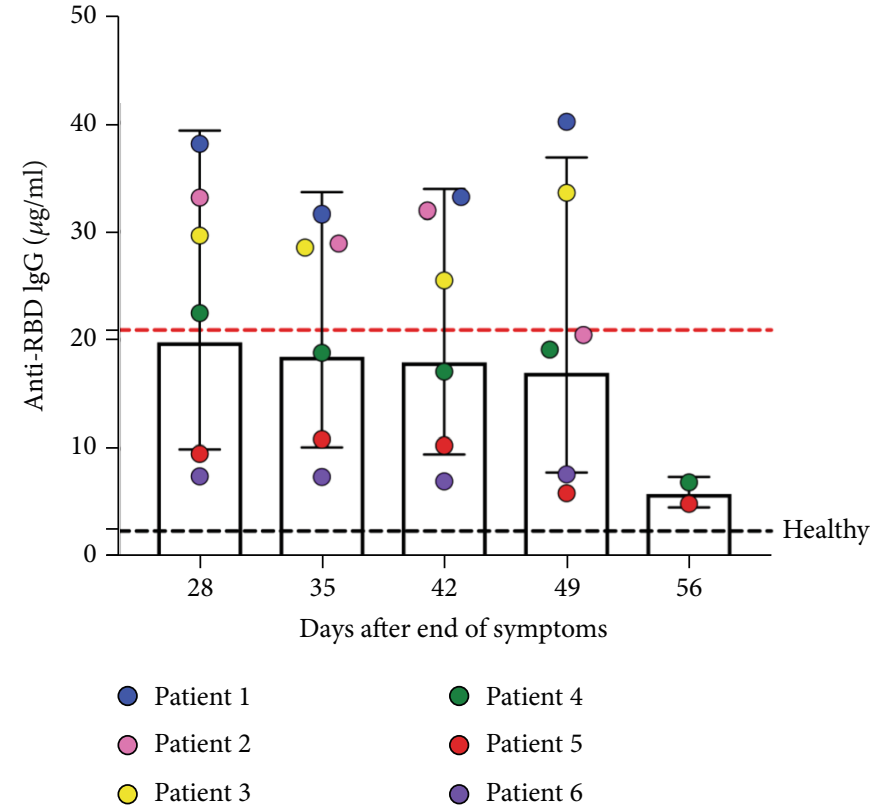

(a)

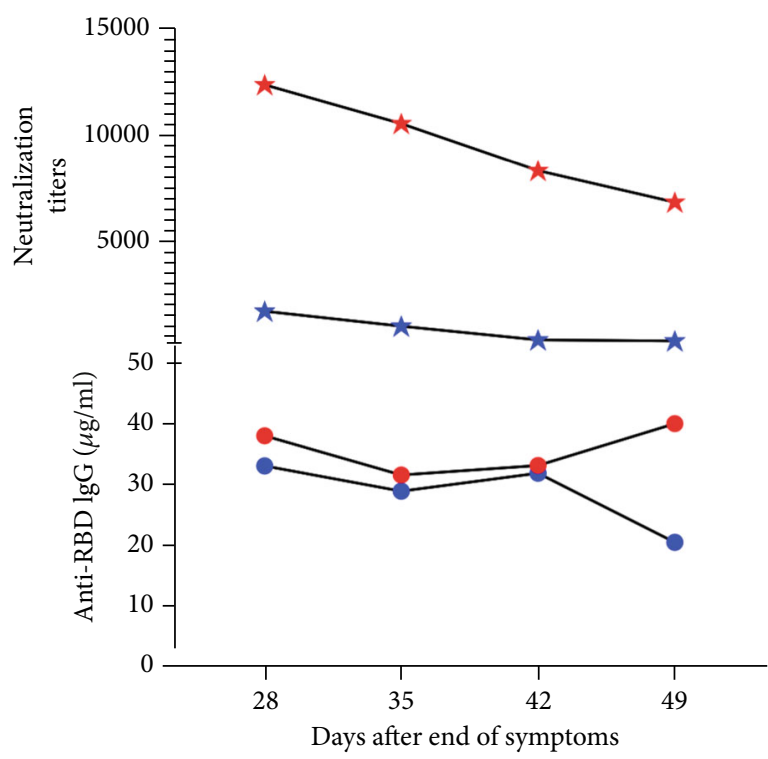

(c)

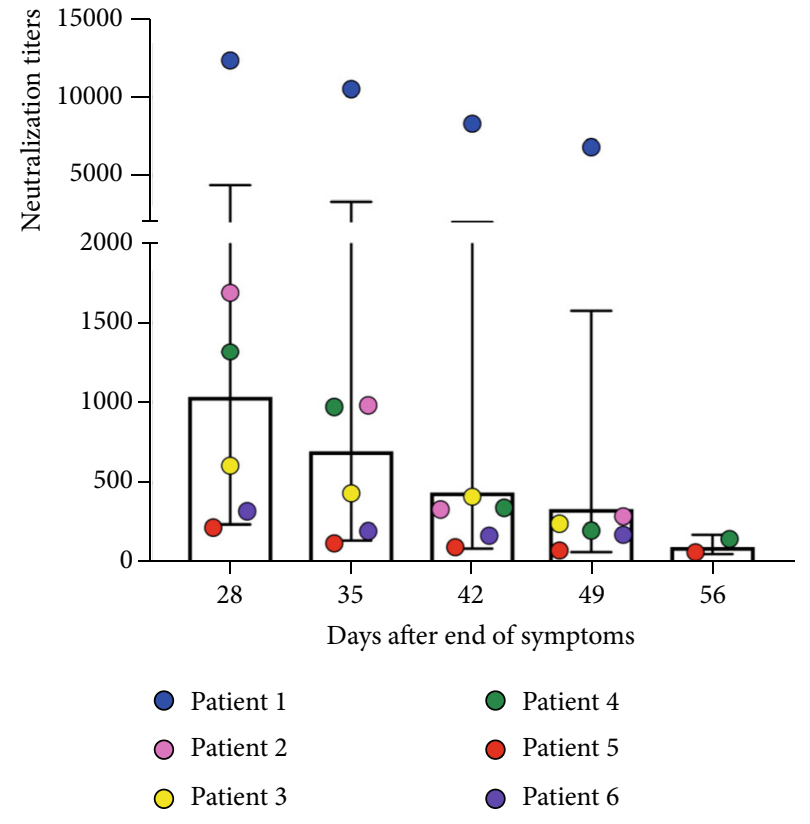

(b)

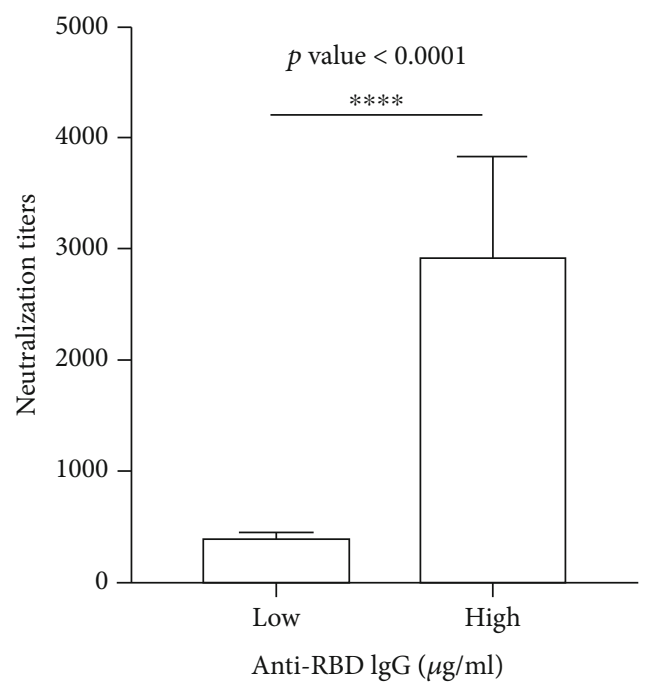

(d)

Anti-RBD lgG

\begin{tabular}{cc|c|c|c}
\multicolumn{2}{c|}{$p$ value $<0.0001$} & Low & High & Total \\
\hline $\begin{array}{c}\text { Neutralization } \\
\text { activity }\end{array}$ & Low & $37(51.39 \%)$ & $23(31.94 \%)$ & 60 \\
\hline Total & High & $0(0 \%)$ & $12(16.67 \%)$ & 12 \\
\hline \multicolumn{2}{c|}{ Tonn } & 37 & 35 & 72
\end{tabular}

(e)

Figure 3: Convalescent plasma donors and IgG antiviral levels. Anti-RBD IgG concentration (a) and neutralizing activity (b) were determined at different days of convalescence. In (c), representative cases with a similar IgG concentration and decreasing neutralizing antibodies. (d) Neutralization activity and IgG levels; average neutralizing activity was represented for stratified high $(N=20)$ and low $(N=52)$ IgG anti-RBD cases based on the 75th percentile. The results are presented as the mean \pm SEM, and statistical differences from the Mann-Whitney test are shown. (e) $2 \times 2$ contingence analysis for high/low antibody levels (P75) and high/low neutralizing activity (median ID80). 75th percentile (P75) and IgG cut-off are indicated in red and black dotted lines, respectively. 


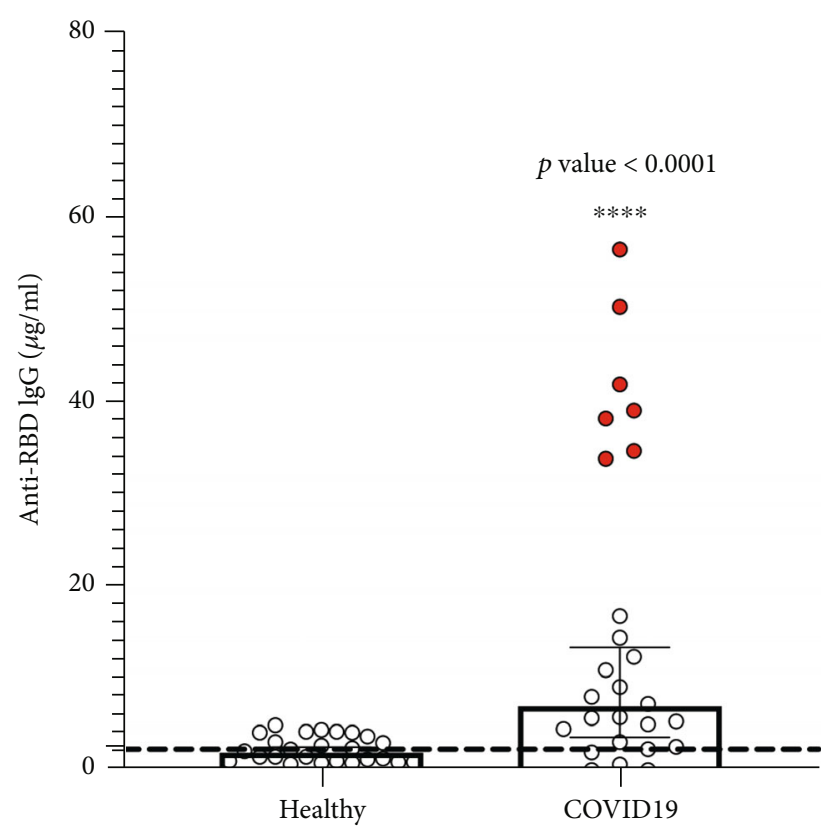

(a)

Anti-RBD $\lg G$

\begin{tabular}{|c|c|c|c|c|}
\hline \multicolumn{2}{|c|}{$N=26$} & Positive & Negative & \multirow{3}{*}{$p$ value $=0.0053$} \\
\hline Days of & First 5 & $5(19.23 \%)$ & $11(42.31 \%)$ & \\
\hline symptom onset & More than 5 & $9(34.62 \%)$ & $1(3.85 \%)$ & \\
\hline \multirow{2}{*}{ Age (years) } & Below 50 & $4(15.38 \%)$ & $3(11.54 \%)$ & \multirow{2}{*}{$p$ value $>0.9999$} \\
\hline & Above 50 & $10(38.46 \%)$ & $9(34.62 \%)$ & \\
\hline \multirow{2}{*}{$\begin{array}{c}\text { Neutralization } \\
\text { activity }\end{array}$} & Low & $3(11.54 \%)$ & $10(38.46 \%)$ & \multirow{2}{*}{$p$ value $=0.0048$} \\
\hline & High & $11(42.31 \%)$ & $2(7.69 \%)$ & \\
\hline
\end{tabular}

Neutralization activity

\begin{tabular}{cc|c|c}
\multicolumn{2}{c}{$N=26$} & High & Low \\
\cline { 1 - 3 } $\begin{array}{c}\text { Days of } \\
\text { symptom onset }\end{array}$ & First 5 & $6(23.08 \%)$ & $10(38.46 \%)$ \\
More than 5 & $7(26.92 \%)$ & $3(11.54 \%)$
\end{tabular}$\quad$ p value $=0.2262$

(b)

FIgURE 4: Anti-RBD IgG antibodies and neutralization activity during active infection with SARS-CoV-2. (a) Anti-RBD IgG levels in participants, namely, healthy controls as the baseline group $(N=25)$ and active COVID-19 patients with different degrees of severity $(N=26)$. (b) $2 \times 2$ contingence analysis for positive/negative antibody levels and days of symptom onset, age (years), neutralization activity and for high/low neutralization activity (median ID80) and days of symptom onset. 75th percentile (P75) is indicated in red circles, and IgG cut-off is indicated by a black dashed line.

anti-RBD-specific seroconversion than hospitalized patients without ICU requirements $(n=35)$ or outpatients with mild to moderate symptoms $(n=86)$. Curiously, at the second week, all the inpatient cases had statistically higher levels of the specific IgG than the outpatient group [22], which was consistent with another report [30].

Typical antibody responses to acute viral infection are quickly induced in COVID-19 patients as shown by Brouwer et al. in 2020, where the seroconversion rate and antibody levels increase rapidly during the first two weeks and the cumulative seropositive rate reaches $50 \%$ on the 11 th day and $100 \%$ on the 39th day [25]. As other authors reported, antibodies against the RBD of spike can be detected at the median of 11 days after the onset of symptoms and the timing of seroconversion may not correlate with the clinical course $[6,8]$.

In 2020, Gozalbo-Rovira showed an anti-RBD IgG threshold above $1.15 \mathrm{AU} / \mathrm{mL}$ as a predictor of neutralizing antibody (NAb) titers, suggesting that quantitative IgG levels may correlate with neutralizing titers with high sensitivity and specificity [27]. In general, more than $70 \%$ of the population included in this study developed anti-RBD IgG 
antibodies, and $24.9 \%$ of the positive cases were above $20.9 \mu \mathrm{g} / \mathrm{mL}$, suggesting a heterogeneous seroconversion but a sustained production of the specific IgG up to 100 days after symptom offset, independenly of age and gender. All convalescent participants showed neutralizing activity with a variability up to 100 times between cases. However, high levels of specific IgG, even though above P75, did not guarantee a significant antiviral or neutralizing activity in plasma. These results were concordant with the results shown by Wang in 2020, where IgG levels (anti-S or anti-N) exhibited a moderate correlation with neutralization titers in plasma (Pearson's $r=0.5393$ and $r=0.6709$, respectively) [32]. A theoretical modeling report suggested that the probability of detecting IgG could reach a maximum of around 25-27 days after COVID-19 symptoms end, with a predicted positivity between $98 \%$ and $100 \%$ of individuals for anti-SARS-CoV-2 IgG [26]. The report also predicted that the probability of detecting NAb in plasma may rapidly rise to near $100 \%$ around 29 days after symptoms end without significant differences between mild/moderate and severe/clinical cases [33]. Previously, a trial using COVID-19 convalescent plasma was halted prematurely because $80 \%$ (53 of 66) of symptomatic participants for only 10 days at the time of enrolment showed seroconversion [27]. In addition, the authors reported SARS-CoV-2 neutralizing activity in $79 \%$ of the plasma receptors before transfusion (44 of 56) with median titers comparable to the donors $(1: 160$ vs. $1: 160$, $p$ $=0.40$ ). Our results have confirmed that most of the Chilean population which recovered from COVID-19 developed anti-SARS-CoV-2 antibodies even only a few days after infection as shown in patients with critical conditions. The quantification of anti-RBD IgG within the local population is necessary as an objective criterion to estimate the neutralizing activity as a protective biomarker within the infected population with SARS-CoV-2. It may be also considered when the laboratory measurement of neutralizing antibodies is limited. As a limitation of this study, the reduced number of cases for some analysis do not allow us to consider our cohort as representative of a larger population. We did not include cases with active disease classified as mild or moderate COVID-19, so we cannot estimate if there is or is not an association between symptomatology and early seroconversion.

\section{Conclusions}

In general, more than $70 \%$ of the population included in this study developed anti-RBD antibodies with a variable neutralizing activity, suggesting a heterogeneous seroconversion after recovering from COVID-19 with a sustained production of specific IgG up to 100 days, independent of age and gender.

\section{Data Availability}

The clinical data used to support the findings of this study are available from the corresponding authors upon request.

\section{Ethical Approval}

This study is part of the clinical trial NCT04384588 (clinicaltrials.gov). This research was reviewed and approved by the Institutional Scientific and Ethical Committee of Instituto Oncológico Fundación Arturo López Pérez (Santiago, Chile). This study complied with all the regulatory authorizations from the Medical Direction together with all the legal and ethical requirements of Chilean law.

\section{Conflicts of Interest}

The authors declare that they have no conflicts of interest.

\section{Authors' Contributions}

Conceptualization was performed by RGS, FVE, and RG. Methodology was selected by RGS, FVE, KC, JLB, CB, AO, SR, FV-E, and RSR. Validation was performed by RGS, FVE, CS, CB, and RSR. Formal analysis was performed by RGS, FVE, $C B$, and RSR. Investigation was performed by RGS, KC, FVE, CB, AO, SR, and JLB. Resources were acquired by RG, JLB, CS, CC, RM, FY, MM, AA, AO, SR, CB, JLB, and CS. Data curation was performed by CS, JLB, and RG. Writing the original draft was performed by RGS and FVE. Writing, reviewing, and editing were performed by RGS, FVE, RG, JLB, CS, CC, RM, FY, MM, AA, CB, JLB, CS, RSR, FV-E, CB, and HM. Visualization was performed by RGS and FVE. Supervision was performed by FVE, RG, CS, FV-E, and RSR. Project administration was performed by FVE, RG, CS, and CC. Funding acquisition was performed by FVE, RG, CC, and HM. All authors have read and approved the final manuscript. R. González-Stegmaier and K. Cereceda have contributed equally to this work.

\section{Acknowledgments}

This study was supported by "Fondo de Adopción tecnológica SIEmpre" sponsored by SOFOFA (Sociedad de Fomento Fabril); CPC Chile (Confederación de la Producción y de Comercio); Ministerio de Ciencia y Tecnología, Conocimiento e Innovación, Chile; and Fundación Arturo López Pérez (FALP), Santiago, Chile. Additionally, the ELISA test design was partially supported by Chile Conicyt Fondecyt Postdoctoral Grant 3170356 (RGS), and the neutralizing antibody assay design was supported by ANID Chile FONDECYT grant Nos. 1190156 (RS-R) and 1180798 (FV-E), CONICYT grant Nos. 21190771 (AA-A) and 21160818 (SR-B), and Proyecto de Internacionalización grant No. UCH-1566 (CB-P).

\section{Supplementary Materials}

Supplementary Figure 1: qualitative and quantitative ELISA performance. In (a), a histogram is shown with the calculated absorbance ratio in a qualitative analysis (positive, ratio above 1.1 as dotted line) and its corresponding anti-RBD IgG concentration range. Data are shown as mean \pm 95 C.I. (b) Correlation between qualitative and quantitative ELISA analysis; average values for absorbance ratio and 
concentration are represented as continuous variables. Supplementary Figure 2: high and low levels of anti-RBD IgG in recovered COVID-19 patients. High $(n=116)$ and low ( $n=350)$ levels were estimated as above or below the 75 th percentile for the global cohort (a) or separated by gender (b). In both cases, the dashed line corresponds to the group of healthy individuals. Asterisks indicate significant differences between groups and analyzed with the MannWhitney test. ${ }^{* * * *} p<0.0001$. (Supplementary Materials)

\section{References}

[1] N. Zhu, D. Zhang, W. Wang et al., "A novel coronavirus from patients with pneumonia in China, 2019," The New England Journal of Medicine, vol. 382, no. 8, pp. 727-733, 2020.

[2] C. Wang, P. W. Horby, F. G. Hayden, and G. F. Gao, "A novel coronavirus outbreak of global health concern," Lancet, vol. 395, no. 10223, pp. 470-473, 2020.

[3] A. L. Phelan, R. Katz, and L. O. Gostin, "The novel coronavirus originating in Wuhan, China," Journal of the American Medical Association, vol. 323, no. 8, pp. 709-710, 2020.

[4] J. S. Weitz, S. J. Beckett, A. R. Coenen et al., "Modeling shield immunity to reduce COVID-19 epidemic spread," Nature Medicine, vol. 26, no. 6, pp. 849-854, 2020.

[5] R. Racine and G. M. Winslow, "IgM in microbial infections: taken for granted?," Immunology Letters, vol. 125, no. 2, pp. 79-85, 2009.

[6] R. Wölfel, V. M. Corman, W. Guggemos et al., "Virological assessment of hospitalized patients with COVID-2019," Nature, vol. 581, no. 7809, pp. 465-469, 2020.

[7] P. Zhou, X. L. Yang, X. G. Wang et al., "A pneumonia outbreak associated with a new coronavirus of probable bat origin," Nature, vol. 579, no. 7798, pp. 270-273, 2020.

[8] J. Zhao, Q. Yuan, H. Wang et al., “Antibody responses to SARS-CoV-2 in patients with novel coronavirus disease 2019," Clinical Infectious Diseases, vol. 71, no. 16, pp. 20272034, 2020.

[9] F. Xiang, X. Wang, X. He et al., "Antibody detection and dynamic characteristics in patients with coronavirus disease 2019," Clinical Infectious Diseases, vol. 71, no. 8, pp. 19301934, 2020.

[10] W. J. Guan, Z. Y. Ni, Y. Hu et al., "Clinical characteristics of coronavirus disease 2019 in China," The New England Journal of Medicine, vol. 382, no. 18, pp. 1708-1720, 2020.

[11] T. Singhal, "A review of coronavirus disease-2019 (COVID19)," Indian Journal of Pediatrics, vol. 87, no. 4, pp. 281-286, 2020.

[12] Q. X. Long, B. Z. Liu, H. J. Deng et al., “Antibody responses to SARS-CoV-2 in patients with COVID-19," Nature Medicine, vol. 26, no. 6, pp. 845-848, 2020.

[13] K. K.-W. To, O. T.-Y. Tsang, W.-S. Leung et al., "Temporal profiles of viral load in posterior oropharyngeal saliva samples and serum antibody responses during infection by SARS-CoV2: an observational cohort study," The Lancet Infectious Diseases, vol. 20, no. 5, pp. 565-574, 2020.

[14] F. Krammer and V. Simon, "Serology assays to manage COVID-19,” Science, vol. 368, no. 6495, pp. 1060-1061, 2020.

[15] O. F. Norheim, "Protecting the population with immune individuals," Nature Medicine, vol. 26, no. 6, pp. 823-824, 2020 .
[16] F. Amanat, D. Stadlbauer, S. Strohmeier et al., "A serological assay to detect SARS-CoV-2 seroconversion in humans," Nature Medicine, vol. 26, no. 7, pp. 1033-1036, 2020.

[17] N. M. A. Okba, M. A. Müller, W. Li et al., "Severe acute respiratory syndrome coronavirus 2-specific antibody responses in coronavirus disease patients," Emerging Infectious Diseases, vol. 26, no. 7, pp. 1478-1488, 2020.

[18] B. Meyer, C. Drosten, and M. A. Müller, "Serological assays for emerging coronaviruses: challenges and pitfalls," Virus Research, vol. 194, pp. 175-183, 2014.

[19] C. Beltrán-Pávez, S. Riquelme-Barrios, A. Oyarzún-Arrau et al., "Insights into neutralizing antibodies responses in individuals exposed to SARS-CoV-2 in Chile. Accepted, Science Advances abe6855," 2020.

[20] L. Guo, L. Ren, S. Yang et al., "Profiling early humoral response to diagnose novel coronavirus disease (COVID-19)," Clinical Infectious Diseases, vol. 71, no. 15, pp. 778-785, 2020.

[21] D. F. Gudbjartsson, G. L. Norddahl, P. Melsted et al., "Humoral immune response to SARS-CoV-2 in Iceland," The New England Journal of Medicine, vol. 383, no. 18, pp. 1724-1734, 2020.

[22] K. Röltgen, A. E. Powell, O. F. Wirz et al., "Defining the features and duration of antibody responses to SARS-CoV-2 infection associated with disease severity and outcome," Science Immunology, vol. 5, no. 54, article eabe0240, 2020.

[23] M. Secchi, E. Bazzigaluppi, C. Brigatti et al., "COVID-19 survival associates with the immunoglobulin response to the SARS-CoV-2 spike receptor binding domain," The Journal of Clinical Investigation, vol. 130, no. 12, pp. 6366-6378, 2020.

[24] L. Li, X. Tong, H. Chen et al., "Characteristics and serological patterns of COVID-19 convalescent plasma donors: optimal donors and timing of donation," Transfusion, vol. 60, no. 8 , pp. 1765-1772, 2020.

[25] P. J. M. T. Brouwer, G. Caniels, K. van der Straten et al., "Potent neutralizing antibodies from COVID-19 patients define multiple targets of vulnerability," Science, vol. 369, no. 6504, pp. 643-650, 2020.

[26] S. L. Klein, A. Pekosz, H. S. Park et al., "Sex, age, and hospitalization drive antibody responses in a COVID-19 convalescent plasma donor population," The Journal of Clinical Investigation, vol. 130, no. 11, pp. 6141-6150, 2020.

[27] R. Gozalbo-Rovira, E. Gimenez, V. Latorre et al., "SARSCoV-2 antibodies, serum inflammatory biomarkers and clinical severity of hospitalized COVID-19 patients," Journal of Clinical Virology : the official publication of the Pan American Society for Clinical Virology, vol. 131, article 104611, 2020.

[28] H. Ma, W. Zeng, H. He et al., "Serum IgA, IgM, and IgG responses in COVID-19," Cellular \& Molecular Immunology, vol. 17, no. 7, pp. 773-775, 2020.

[29] J. Sun, X. Tang, R. Bai et al., "The kinetics of viral load and antibodies to SARS-CoV-2," Clinical Microbiology and Infection : the official publication of the European Society of Clinical Microbiology and Infectious Diseases, vol. 26, no. 12, pp. 1690.e1-1690.e4, 2020.

[30] E. Marklund, S. Leach, H. Axelsson et al., "Serum-IgG responses to SARS-CoV-2 after mild and severe COVID-19 infection and analysis of IgG non-responders," PLoS One, vol. 15, no. 10, article e0241104, 2020.

[31] A. Padoan, L. Sciacovelli, D. Basso et al., "IgA-Ab response to spike glycoprotein of SARS-CoV-2 in patients with COVID-19: 
a longitudinal study," Clinica Chimica Acta, vol. 507, pp. 164166, 2020.

[32] Y. Wang, L. Zhang, L. Sang et al., "Kinetics of viral load and antibody response in relation to COVID-19 severity," The Journal of Clinical Investigation, vol. 130, no. 10, pp. 52355244, 2020.

[33] B. Borremans, A. Gamble, K. C. Prager et al., "Quantifying antibody kinetics and RNA detection during early-phase SARS-CoV-2 infection by time since symptom onset," eLife, vol. 9, article e60122, 2020.

[34] K. Cereceda, R. González-Stegmaier, J. L. Briones et al., Serological Profile of Specific Antibodies against Dominant Antigens of SARS-CoV-2 in Chilean COVID-19 Patients, Unpublished, Submitted PlosOne, 2020. 